\title{
Factors associated with retro-odontoid soft-tissue thickness in rheumatoid arthritis
}

\author{
Sho Dohzono, MD, PhD, Akinobu Suzuki, MD, PhD, Tatsuya Koike, MD, PhD, \\ Shinji Takahashi, MD, PhD, Kentaro Yamada, MD, PhD, Hiroyuki Yasuda, MD, PhD, and \\ Hiroaki Nakamura, MD, PhD
}

Department of Orthopaedic Surgery, Osaka City University Graduate School of Medicine, Osaka, Japan

\begin{abstract}
OBJECTIVE Increasing soft-tissue mass posterior to the odontoid process causes spinal cord compression. Retroodontoid pseudotumors are considered to be associated with atlantoaxial instability in patients with rheumatoid arthritis (RA), but the exact mechanism by which these lesions develop has not been elucidated. The purpose of this study was to identify the relationships between retro-odontoid soft-tissue (ROST) thickness and radiological findings or clinical data in patients with RA.
\end{abstract}

METHODS A total of 201 patients with RA who had been followed up at the outpatient clinic of the authors' institution were enrolled in this study. ROST thickness was evaluated on midsagittal T1-weighted MRI. The correlations between ROST thickness and radiographic findings or clinical data on RA were examined. The independent factors related to ROST thickness were analyzed using stepwise multiple regression analysis.

RESULTS The average thickness of ROST was $3.0 \pm 1.4 \mathrm{~mm}$. ROST thickness showed an inverse correlation with disease duration $(r=-0.329, p<0.01)$, Steinbrocker stage $(r=-0.284, p<0.01)$, the atlantodental interval (ADI) in the neutral position $(r=-0.326, p<0.01)$, the ADI in the flexion position $(r=-0.383, p<0.01)$, and the ADI in the extension position $(r=-0.240, p<0.01)$. On stepwise multiple regression analysis, ADI in the flexion position and Steinbrocker stage were independent factors associated with ROST thickness.

CONCLUSIONS Although the correlations were not strong, ROST thickness in patients with RA was inversely correlated with ADI and Steinbrocker stage. In other words, ROST thickness tends to be smaller as atlantoaxial instability and peripheral joint destruction worsen.

Clinical trial registration no.: UMIN000000980 (UMIN Clinical Trials Registry)

http://thejns.org/doi/abs/10.3171/2016.3.SPINE15787

KEY WORDS rheumatoid arthritis; magnetic resonance imaging; retro-odontoid pseudotumor; atlantoaxial instability; odontoid process; transverse ligament; cervical spine

I NCREASED soft tissue mass in the upper cervical region posterior to the odontoid process, so-called "retroodontoid pseudo tumor," can cause spinal cord compression. Retro-odontoid pseudotumor is most often observed in patients with rheumatoid arthritis (RA), and it is considered to be associated with atlantoaxial instability ${ }^{11,13,27}$ or synovial inflammation. ${ }^{19}$ Nevertheless, retroodontoid pseudotumor secondary to RA is not a common condition, even though atlantoaxial subluxation is often seen in patients with RA.
Moreover, some reports have shown that retro-odontoid pseudotumor can develop without apparent atlantoaxial joint instability in patients without inflammatory disease. ${ }^{3,5}$ Mechanical stress due to subaxial spinal fusion and repetitive transverse ligament damage has been thought to be the main cause of the pseudotumor,, 314 but few studies have investigated the factors related to pseudotumor, including atlantoaxial motion, and the exact mechanism by which retro-odontoid pseudotumors develop has not been elucidated. The purpose of this study was to identify the

ABBREVIATIONS $A D I=$ atlantodental interval; $I C C=$ intraclass correlation coefficient; $m H A Q=$ modified Health Assessment Questionnaire; $R A=$ rheumatoid arthritis; ROST = retro-odontoid soft tissue. 
relationships between retro-odontoid soft-tissue (ROST) thickness and radiological findings and clinical data in patients with RA.

\section{Methods}

From August 2010 to June 2011, a total of 234 patients with RA who had been followed up at the outpatient clinic of our institution were recruited into a cross-sectional study. All patients fulfilled the American Rheumatism Association's 1987 criteria for the classification of RA. ${ }^{2}$ Patients with previous cervical spine surgery were excluded. Clinical information on RA, symptoms related to the cervical spine, and radiological examination results were collected. The study protocol was approved by the Ethics Committee for Clinical Research at Osaka City University School of Medicine. The study was registered with the University Hospital Medical Information Network Clinical Trials Registry (no. UMIN000000980). The current article presents the results of a subanalysis of data collected for that study.

\section{Clinical Evaluation}

Clinical data on RA included disease duration, functional class, and the 28-joint Disease Activity Score using the erythrocyte sedimentation rate (DAS28 ESR) ${ }^{16}$ at enrollment. Functional class was assessed by the modified Health Assessment Questionnaire (mHAQ) ${ }^{15}$ Neurological symptoms were evaluated using the Ranawat classification (Grades I, II, IIIA, and IIIB). ${ }^{18}$

Plain posteroanterior radiographs of bilateral wrists and hands were used to evaluate the severity of rheumatoid joint destruction according to the Steinbrocker classification (Stage I-IV) ${ }^{21}$ The atlantodental interval (ADI), Ranawat value, and sagittal Cobb angle between C-2 and C-7 were measured on lateral views. ADIs were independently evaluated on images in the flexion, neutral, and extension positions.

\section{MRI Evaluation}

All patients underwent MRI with the same 3-T unit (Achieva 3.0 T, Philips). ROST thickness was estimated on midsagittal T1-weighted images as previously reported (Fig. 1). ${ }^{23}$

\section{Statistical Analysis}

IBM SPSS Statistics, version 19 (IBM Corp.) was used for all statistical analyses. To evaluate the reliability of techniques used to measure ROST, we evaluated the intra- and interobserver variabilities. The parameters were measured independently by 2 spine surgeons (S.D. and S.T.), both authors with more than 10 years of experience, who were blinded to the patients' clinical variables. After each surgeon had measured a parameter on a radiograph twice, the inter- and intraobserver variability were evaluated by means of the intraclass coefficient of correlation (ICC).

Correlation between ROST thickness and age, disease duration, DAS28 ESR, mHAQ, Steinbrocker stage, neurological symtpoms according to the Ranawat classifica-

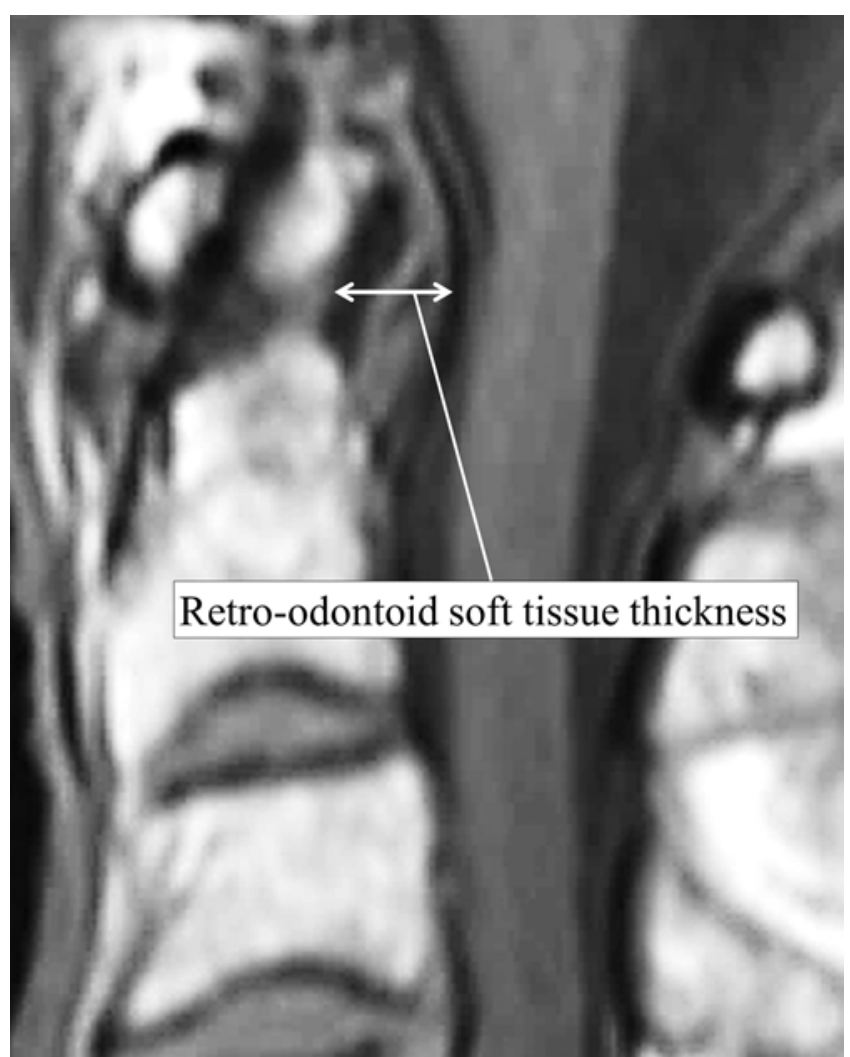

FIG. 1. Midsagittal T1-weighted MR image showing measurement of ROST.

tion, ADI (neutral, flexion, and extension), and Ranawat value was assessed using the Pearson product moment correlation coefficient (for continuous/numerical data) or the Spearman rank correlation coefficient (for categorical data). Variables with a correlation coefficient value greater than 0.7 were defined as confounding factors. Any variable with a statistically significant finding $(\mathrm{p}<0.05)$ was selected as a candidate for a stepwise multiple regression analysis to evaluate the factors independently associated with ROST thickness in RA patients after adjustment for confounding factors.

\section{Results}

Of the 234 patients who were recruited, 33 were excluded from the study. These patients were excluded for the following reasons: presence of other somatic disease $(\mathrm{n}=2)$, contraindications to MRI $(\mathrm{n}=2)$, prior cervical spine surgery $(n=3)$, declining to undergo MRI $(n=9)$, and declining to participate in this study $(\mathrm{n}=17)$. The remaining 201 patients constituted the study group. Baseline demographic and clinical data at the time of recruitment are shown in Table 1.

We confirmed the reliability of the radiographic measurements of ROST thickness by calculating intra- and interobserver variabilities of the parameters. The intraobserver ICC values were more than 0.98 for both observers, and the interobserver ICC was 0.966 , indicating excellent reproducibility of the measurements.

The average thickness of ROST was $3.0 \pm 1.4 \mathrm{~mm}$. A 
TABLE 1. Demographic and clinical characteristics

\begin{tabular}{lc}
\hline \multicolumn{1}{c}{ Characteristic } & Value \\
\hline Female sex & $166(83)$ \\
\hline Mean age (yrs) & $62.3 \pm 10.7$ \\
\hline RA duration (yrs) & $13.5 \pm 11.8$ \\
\hline Steinbrocker stage & \\
\hline I & $44(22)$ \\
\hline II & $53(27)$ \\
\hline II & $68(34)$ \\
\hline IV & $36(28)$ \\
\hline DAS28 ESR & $3.5(1.3)$ \\
\hline mHAQ score & $3.6(4.7)$ \\
\hline VAS (neck pain) & $20.0(22.4)$ \\
\hline Neurological symptoms (Ranawat classification) & \\
\hline Class I & $155(77)$ \\
\hline Class II & $41(22)$ \\
\hline Class IIla & $5(2)$ \\
\hline Class IIIb & 0 \\
\hline Radiographic findings & \\
\hline ADI in the neutral position & $2.2(1.7)$ \\
\hline ADI in the flexion position & $3.0(2.2)$ \\
\hline ADI in the extension position & $1.6(1.1)$ \\
\hline Ranawat value & $16.5(2.6)$ \\
\hline
\end{tabular}

VAS = visual analog scale.

frequency histogram of ROST is shown in Fig. 2. The thickness showed an inverse correlation with disease duration $(r=-0.329, p<0.01)$, Steinbrocker stage $(r=-0.284$, $\mathrm{p}<0.01)$, ADI in the neutral position $(r=-0.326, p<0.01)$, ADI in the flexion position ( $\mathrm{r}=-0.383, \mathrm{p}<0.01)$, and ADI in the extension position $(\mathrm{r}=-0.240, \mathrm{p}<0.01)$. There was no statistically significant correlation between ROST
TABLE 2. Correlations with ROST thickness

\begin{tabular}{lrr}
\hline \multicolumn{1}{c}{ Variable } & $r$ & $p$ Value \\
\hline Patient age & -0.074 & 0.294 \\
\hline Disease duration & -0.329 & $<0.001$ \\
\hline DAS28 ESR & -0.159 & 0.027 \\
\hline mHAQ score & -0.194 & 0.007 \\
\hline Steinbrocker stage & -0.284 & $<0.001$ \\
\hline ADI (neutral) & -0.326 & $<0.001$ \\
\hline ADI (flexion) & -0.383 & $<0.001$ \\
\hline ADI (extension) & -0.240 & 0.001 \\
\hline Ranawat value & 0.254 & $<0.001$ \\
\hline Cobb angle btwn C-2 \& C-7 (sagittal) & -0.049 & 0.496 \\
\hline
\end{tabular}

thickness and age, DAS28 ESR, or mHAQ score (Table 2). Stepwise multiple regression analysis showed that ADI in the flexion position and Steinbrocker stage were independently associated with ROST thickness (Table 3). A scatter diagram that shows ADI in the flexion position and ROST thickness is presented as Fig. 3.

ROST thicknesses tended to be greater when the ADI was short and smaller when the ADI was long. The inverse correlation between ROST thickness and the ADI can be seen in the representative cases shown in Figs. 4 and 5.

\section{Discussion}

In the present study, ROST thickness was found to decrease with atlantoaxial instability in patients with RA. Many clinical studies have suggested that mechanical stress on the transverse ligament causes retro-odontoid pseudotumor, ${ }^{5,10,13,22}$ but the present results suggest that atlantoaxial instability is associated with decreased stress on the transverse ligament.

Anatomically, the transverse ligament of the atlas runs

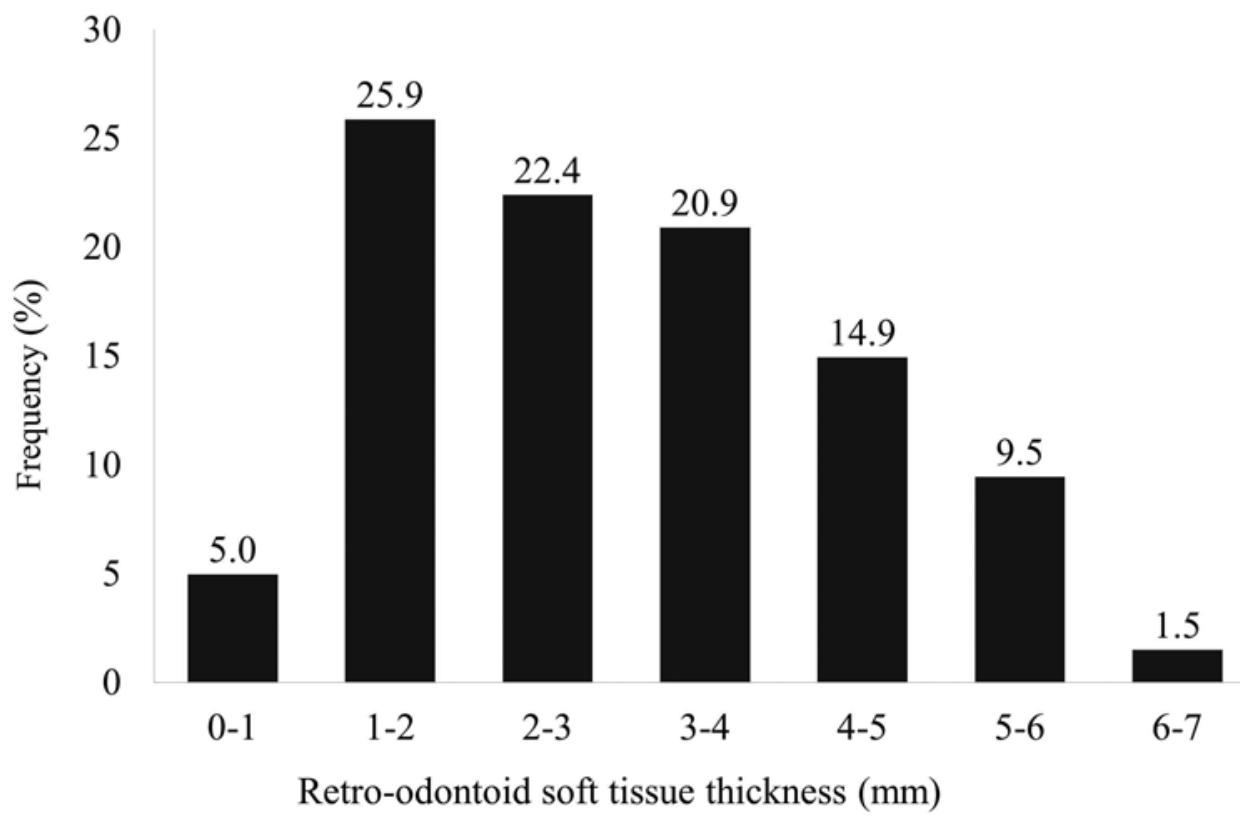

FIG. 2. Distribution of ROST thickness in RA patients. 


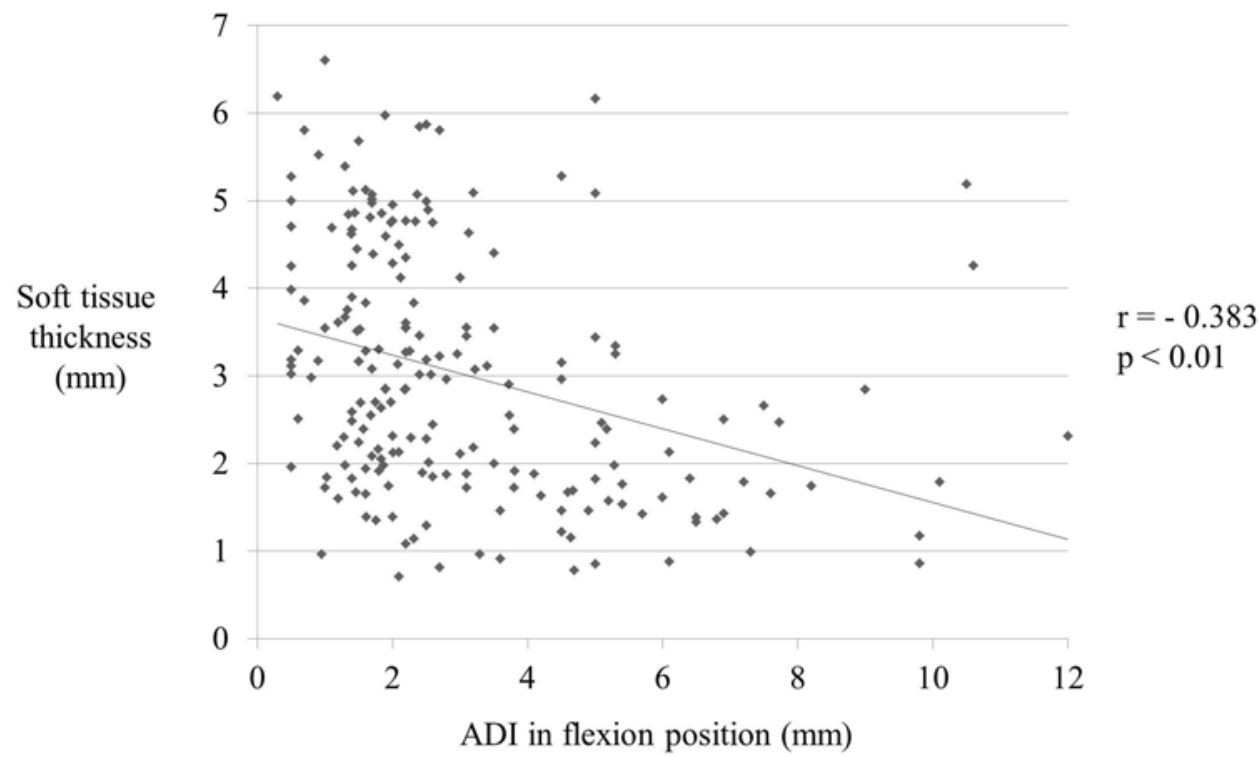

FIG. 3. Correlation between retro-odontoid soft tissue thickness and the ADI in the flexion position.

posterior to the dens and attaches to the lateral tubercles of the atlas bilaterally. There is a synovial capsule between the odontoid process and the transverse ligament. The tectorial membrane and epidural fat are located dorsal to the transverse ligament. ${ }^{6}$ Therefore, ROST consists of synovial capsule, transverse ligament, and tectorial membrane. ${ }^{24}$

The transverse ligament, which is the main component of ROST, shows low signal intensity on T1- and T2weighted MRI. Retro-odontoid pseudotumors in patients without inflammatory disease show low signal intensity on both T1- and T2-weighted MRI. ${ }^{22,24}$ On the other hand, retro-odontoid pseudotumors in patients with RA are typically visualized as being hypo- to iso-intense on T1 and hyperintense on T2-weighted MRI. ${ }^{7,10-12,27}$ Yonezawa et al., ${ }^{25}$ however, reported 3 types of retro-odontoid masses on MRI in patients with RA: pannus type (T1 low, T2 high), pseudotumor type (T1 low, T2 low), and mixed type (T1 low, T2 low and high). High signal intensity on T2-weighted images indicates that the pseudotumor consists of an increased amount of water, as in inflammatory tissue, and low signal intensity T2-weighted images indicates that the pseudotumor contains fibrous material. ${ }^{11,22}$

With regard to pathology, Sze et al. ${ }^{22}$ reported hypertrophy of fibrocartilage tissue in patients without inflammatory disease, and Oohori et al. ${ }^{13}$ reported that the retro-odontoid mass in an RA patient was composed of degenerated fibrocartilaginous tissue and was predomi-

TABLE 3. Factors associated with ROST thickness on stepwise multiple regression analysis*

\begin{tabular}{lccccc}
\hline \multicolumn{1}{c}{ Variable } & B & SE (B) & Beta & $t$ & $p$ Value† \\
\hline ADI (flexion) & -0.169 & 0.044 & -0.268 & -3.798 & $<0.001$ \\
\hline Steinbrocker stage & -0.261 & 0.096 & -0.191 & -2.708 & 0.007 \\
\hline
\end{tabular}

SE $=$ standard error.

* $R=0.380, r^{2}=0.145$, adjusted $r^{2}=0.136$.

$\dagger$ For $t$. nantly necrotic. However, there has been no report on the association between pathological and MRI findings in retro-odontoid pseudotumors.

As for the pathogenesis of retro-odontoid pseudotumor, there have been reports that the lesion is related to atlantoaxial instability in RA patients. ${ }^{10,22}$ Grob et al.$^{10}$ reported that retro-odontoid pseudotumor was maintained by motion rather than by the inflammatory process itself. The fact that spontaneous mass reduction occurred after spinal fusion supports the hypothesis that retro-odontoid pseudotumor is related to instability or mechanical stress on the atlantoaxial joint. Similarly, the current study showed that ROST thickness was not correlated with DAS28 ESR, which represents RA disease activity. Sono et al. ${ }^{17}$ reported on 5 patients with RA and 6 patients without RA who underwent surgery due to retro-odontoid pseudotumor. They reported that atlantoaxial subluxation was found in all RA patients with a retro-odontoid pseudotumor.

The current study, however, showed that ROST thick-
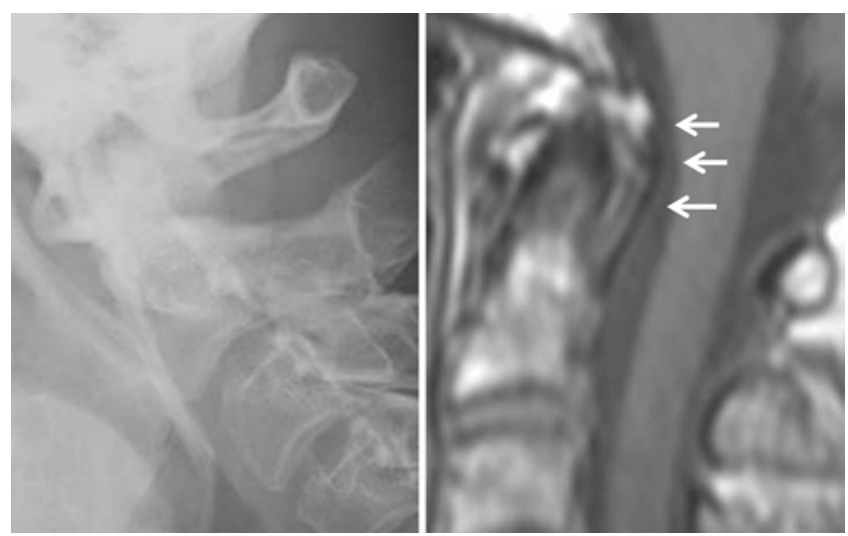

FIG. 4. Representative Case 1: large ROST thickness and short ADI. This 64-year-old woman had a 14 -year history of RA. The ADI on the lateral flexion radiograph (left) was $1.4 \mathrm{~mm}$, while the ROST thickness on the midsagittal T1-weighted MR image (right) was $4.3 \mathrm{~mm}$. The arrows indicate ROST. 

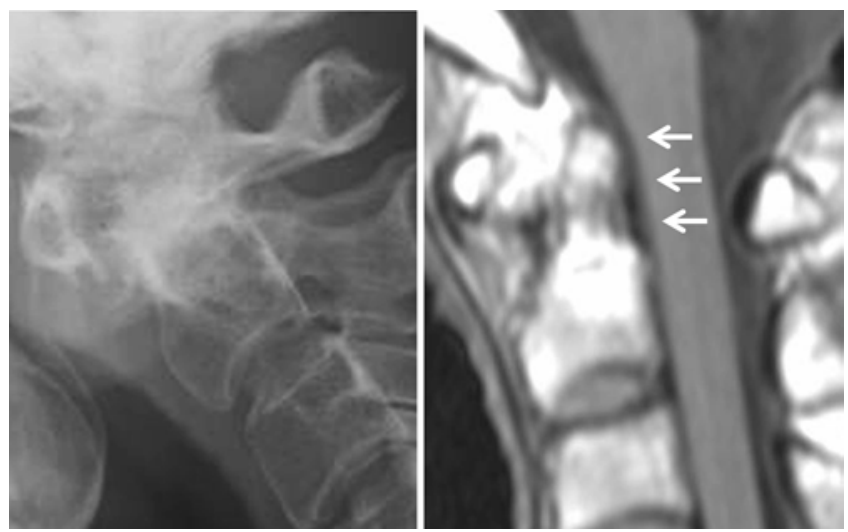

FIG. 5. Representative Case 2: smaller ROST thickness and long ADI. This 62-year-old woman had an 8-year history of RA. The ADI on the lateral flexion radiograph (left) was $6.8 \mathrm{~mm}$, while the ROST thickness on the midsagittal T1-weighted MR image (right) was $1.4 \mathrm{~mm}$. The arrows indicate ROST

ness tended to be smaller when the ADI was large. This finding indicates a different view of the formation of retroodontoid masses in patients with RA. The biomechanical data reported by Fielding et al. ${ }^{9}$ demonstrated that the transverse ligament only allows anterior subluxation of the atlas of about 3-5 $\mathrm{mm}$ before rupturing. This means that, when the ADI is more than $3-5 \mathrm{~mm}$, the stress to the transverse ligament would decrease. While the ADI is less than $3-5 \mathrm{~mm}$, the remaining transverse ligament would thicken by the mechanical stress. Therefore, atlantoaxial instability should be considered a result of the degeneration process, not a prerequisite for the formation of the retro-odontoid mass. Atlantoaxial subluxation is commonly seen in patients with RA, and its prevalence in these patients ranges from $16 \%$ to $50 \%$ according to previous reports involving more than 200 patients. ${ }^{1,4,20,23,26}$ On the other hand, there are few reports about retro-odontoid pseudotumor secondary to RA. These facts imply that retro-odontoid pseudotumor is less associated with atlantoaxial instability.

Tojo et al. ${ }^{24}$ studied ROST thickness in a series of 503 cervical spine MRI studies. They concluded that there was no association between ROST thickness and the presence of RA. However, the radiological and clinical assessments in their study were limited. First, the ADI was not evaluated. Second, the history of RA was determined only from patient medical records, and no clinical assessment was performed. In the present study, we showed that ROST thickness was inversely correlated with the ADI in the flexion position and with Steinbrocker stage. Taking the present results and previous reports into consideration, we suspect that ROST thickness is related to mechanical stress but not the degree of motion. Possible explanations for these findings are 1) that C1-2 joint inflammation or destruction causes increase of mechanical stress at the transverse ligament followed by increased ROST, 2) that more stress causes stretching or tearing of the transverse ligament as RA progresses, 3) that mechanical stress to the transverse ligament might decrease, and 4) that as a result, the transverse ligament might shrink and ROST decrease.

There are several limitations to the present study. First, the present analysis was the subanalysis of the imaging data collected in the past. Although the therapy using biologics had already spread at the time of data collection (36\% of patients in this study were treated with biologics), long-term medication with biologic agents or therapy with newer biologics is not reflected in the present results. The influence of biologics on the retro-odontoid mass should be investigated again in the future. The present sample, however, was sufficient to yield substantial effects. Power analysis using software (G*power 3.1.9.2 Erdfelder, Faul, \& Buchner, 1996) ${ }^{8}$ showed that the total sample size needed was about 100 patients for linear multiple regression to obtain sufficient power (0.8), with medium effect size, an $\alpha$ error probability of 0.05 , and 6 predictor variables. Second, no cases of surgically treated retro-odontoid pseudotumor were included. It remains unclear whether pseudotumors are pathologically similar to ROST thickness. Despite these limitations, this was the largest study to analyze ROST thickness in patients with RA and the first to show that atlantoaxial subluxation might decrease stress on ROST.

\section{Conclusions}

Although the correlations were not strong, ROST thickness in patients with RA was inversely correlated with ADI and Steinbrocker stage. In other words, ROST thickness tends to be smaller as atlantoaxial instability and peripheral joint destruction worsen.

\section{Acknowledgments}

We would like to thank A. Kamiyama, Dr. M. Tada, Y. Sugioka, T. Okano, K. Tsukiyama, and Y. Shinohara for assistance with data collection.

\section{References}

1. Ahn JK, Hwang JW, Oh JM, Lee J, Lee YS, Jeon CH, et al: Risk factors for development and progression of atlantoaxial subluxation in Korean patients with rheumatoid arthritis. Rheumatol Int 31:1363-1368, 2011

2. Arnett FC, Edworthy SM, Bloch DA, McShane DJ, Fries JF, Cooper NS, et al: The American Rheumatism Association 1987 revised criteria for the classification of rheumatoid arthritis. Arthritis Rheum 31:315-324, 1988

3. Chikuda H, Seichi A, Takeshita K, Shoda N, Ono T, Matsudaira K, et al: Radiographic analysis of the cervical spine in patients with retro-odontoid pseudotumors. Spine (Phila Pa 1976) 34:E110-E114, 2009

4. Conlon PW, Isdale IC, Rose BS: Rheumatoid arthritis of the cervical spine. An analysis of 333 cases. Ann Rheum Dis 25:120-126, 1966

5. Crockard HA, Sett P, Geddes JF, Stevens JM, Kendall BE, Pringle JA: Damaged ligaments at the craniocervical junction presenting as an extradural tumour: a differential diagnosis in the elderly. J Neurol Neurosurg Psychiatry 54:817-821, 1991

6. Dickman CA, Mamourian A, Sonntag VK, Drayer BP: Magnetic resonance imaging of the transverse atlantal ligament for the evaluation of atlantoaxial instability. J Neurosurg 75:221-227, 1991

7. Eleraky MA, Masferrer R, Sonntag VK: Posterior atlantoaxial facet screw fixation in rheumatoid arthritis. J Neurosurg 89:8-12, 1998 
8. Faul F, Erdfelder E, Buchner A, Lang AG: Statistical power analyses using $G^{*}$ Power 3.1: tests for correlation and regression analyses. Behav Res Methods 41:1149-1160, 2009

9. Fielding JW, Cochran GVB, Lawsing JF III, Hohl M: Tears of the transverse ligament of the atlas. A clinical and biomechanical study. J Bone Joint Surg Am 56:1683-1691, 1974

10. Grob D, Würsch R, Grauer W, Sturzenegger J, Dvorak J: Atlantoaxial fusion and retrodental pannus in rheumatoid arthritis. Spine (Phila Pa 1976) 22:1580-1584, 1997

11. Larsson EM, Holtås S, Zygmunt S: Pre- and postoperative MR imaging of the craniocervical junction in rheumatoid arthritis. AJR Am J Roentgenol 152:561-566, 1989

12. Milbrink J, Nyman R: Posterior stabilization of the cervical spine in rheumatoid arthritis: clinical results and magnetic resonance imaging correlation. J Spinal Disord 3:308-315, 1990

13. Oohori Y, Seichi A, Kawaguchi H, Tajiri Y, Oda H, Nakamura K: Retroodontoid pseudotumor resected by a high cervical lateral approach in a rheumatoid arthritis patient: a case report. J Orthop Sci 9:90-93, 2004

14. Patel NP, Wright NM, Choi WW, McBride DQ, Johnson JP: Forestier disease associated with a retroodontoid mass causing cervicomedullary compression. J Neurosurg 96 (2 Suppl):190-196, 2002

15. Pincus T, Summey JA, Soraci SA Jr, Wallston KA, Hummon NP: Assessment of patient satisfaction in activities of daily living using a modified Stanford Health Assessment Questionnaire. Arthritis Rheum 26:1346-1353, 1983

16. Prevoo ML, van 't Hof MA, Kuper HH, van Leeuwen MA, van de Putte LB, van Riel PL: Modified disease activity scores that include twenty-eight-joint counts. Development and validation in a prospective longitudinal study of patients with rheumatoid arthritis. Arthritis Rheum 38:44-48, 1995

17. Sono T, Onishi E, Matsushita M: Radiographic risk factors and surgical outcomes for retroodontoid pseudotumors. J Spinal Disord Tech 27:E193-E198, 2013

18. Ranawat CS, O'Leary P, Pellicci P, Tsairis P, Marchisello P, Dorr L: Cervical spine fusion in rheumatoid arthritis. J Bone Joint Surg Am 61:1003-1010, 1979

19. Salli A, Sahin N, Paksoy Y, Kucuksarac S, Ugurlu H: Treatment of periodontoid pannus with infliximab in a patient with rheumatoid arthritis. J Clin Rheumatol 15:250-251, 2009

20. Smith PH, Benn RT, Sharp J: Natural history of rheumatoid cervical luxations. Ann Rheum Dis 31:431-439, 1972

21. Steinbrocker O, Traeger CH, Batterman RC: Therapeutic criteria in rheumatoid arthritis. J Am Med Assoc 140:659-662, 1949

22. Sze G, Brant-Zawadzki MN, Wilson CR, Norman D, Newton TH: Pseudotumor of the craniovertebral junction associated with chronic subluxation: MR imaging studies. Radiology 161:391-394, 1986

23. Takahashi S, Suzuki A, Koike T, Yamada K, Yasuda H, Tada $\mathrm{M}$, et al: Current prevalence and characteristics of cervical spine instability in patients with rheumatoid arthritis in the era of biologics. Mod Rheumatol 24:904-909, 2014

24. Tojo S, Kawakami R, Yonenaga T, Hayashi D, Fukuda K: Factors influencing on retro-odontoid soft-tissue thickness: analysis by magnetic resonance imaging. Spine (Phila Pa 1976) 38:401-406, 2013

25. Yonezawa I, Okuda T, Won J, Sakoda J, Nakahara D, Nojiri $\mathrm{H}$, et al: Retrodental mass in rheumatoid arthritis. J Spinal Disord Tech 26:E65-E69, 2013

26. Yurube T, Sumi M, Nishida K, Takabatake M, Kohyama K, Matsubara T, et al: Progression of cervical spine instabilities in rheumatoid arthritis: a prospective cohort study of outpatients over 5 years. Spine (Phila Pa 1976) 36:647-653, 2011

27. Zygmunt S, Säveland H, Brattström H, Ljunggren B, Larsson EM, Wollheim F: Reduction of rheumatoid periodontoid pannus following posterior occipito-cervical fusion visualised by magnetic resonance imaging. Br J Neurosurg 2:315-320, 1988

\section{Disclosures}

Author T. Koike has received research grants and/or speaking fees from Takeda Pharmaceutical, Mitsubishi Tanabe Pharma Corporation, Chugai Pharmaceutical, Eisai, Abbott Japan, Teijin Pharma, Banyu Pharmaceutical, and Ono Pharmaceutical.

\section{Author Contributions}

Conception and design: Suzuki, Dohzono. Acquisition of data: Suzuki, Dohzono, Koike, Takahashi, Yamada, Yasuda. Analysis and interpretation of data: Dohzono. Drafting the article: Dohzono. Critically revising the article: Suzuki. Reviewed submitted version of manuscript: Suzuki, Dohzono. Approved the final version of the manuscript on behalf of all authors: Suzuki. Statistical analysis: Dohzono, Takahashi. Administrative/technical/material support: Koike. Study supervision: Nakamura.

\section{Correspondence}

Akinobu Suzuki, Department of Orthopaedic Surgery, Osaka City University Graduate School of Medicine, 1-4-3 Asahimachi, Abeno-ku, Osaka 545-8585, Japan. email: a-suzuki@msic.med. osaka-cu.ac.jp. 